\title{
Beyond decision! Motor contribution to speed-accuracy trade-off in decision-making
}

\author{
Laure Spieser $^{1,2,3}$ • Mathieu Servant ${ }^{1,2,4}$ - Thierry Hasbroucq ${ }^{1,2}$ - Borís Burle B $^{1,2}$
}

Published online: 18 October 2016

(C) Psychonomic Society, Inc. 2016

\begin{abstract}
Both in real life and experimental settings, increasing response speed typically leads to more errorprone actions. Processes underlying such a "speed-accuracy trade-off" (SAT) are usually assumed to be purely decisional: cautiousness would be determined only by the amount of sensory evidence required to select a response. The present data challenges this largely accepted view, by directly showing that motor processes are speeded up under time pressure. In a choice reaction time task where emphasis was put either on response speed or accuracy, motor processes were investigated through the analysis of muscular activity related to response execution. When response speed was emphasized, the time between electromyographic onset and behavioral response (motor time) was also speeded up (contributing to more than $20 \%$ of the total effect on global reaction time). This speeded execution (likely due
\end{abstract}

Borís Burle

boris.burle@univ-amu.fr

1 Aix-Marseille Univ, CNRS, LNC UMR 7291, Marseille, France

2 CNRS, Fédération 3C, Aix Marseille Univ, Marseille, France

3 Department of Psychology, City University London, London, UK

4 Department of Psychology, Vanderbilt University, Nashville, TN 3720, USA to a more efficient motor command) may also explain why participants are less able to interrupt incorrect response execution once started (Burle et al., Psychonomic Bulletin \& Review, 21(4), 1003-1010, 2014), leading to more overt errors. Pointing to a speed-accuracy exchange within motor processes themselves, the present results call for a re-evaluation of widely accepted assumptions about SAT, and more generally, decision-making processes. They are discussed in the context of recent extensions of the drift diffusion model framework, questioning the strict separation between decisional and motor processes.

Keywords Decision-making - Speed-accuracy trade-off . Electrophysiology

In everyday life, the accuracy of a decision largely depends on its speed: fast decisions tend to be less accurate than slower more thoughtful ones (Heitz, 2014). Such a "speedaccuracy trade-off" (SAT) is pervasive, being present in a wide range of species, from Drosophila (DasGupta et al., 2014) to humans and in virtually all cognitive abilities (see Heitz, 2014 for an overview). Understanding how displacements along the speed-accuracy trade-off are achieved is of major importance for the comprehension of decision-making processes, and information processing in general. Lately, SAT has received renewed interest thanks to monkey neurophysiology data in relation to sequential sampling models, conceptualizing decision-making as an accumulation of evidence in favor of competing alternatives (Brunton et al., 2013; Heitz \& Schall, 2012; Ratcliff $\&$ Smith, 2004): A decision is made as soon as the amount of evidence accumulated for one of the alternatives has reached a decision threshold (Ratcliff \& Smith, 2004). Varying the distance between accumulation starting point and 
decision threshold naturally accounts for SAT: A high decision threshold requires more evidence and hence leads to slow but more accurate responses, while a low decision threshold results in premature, more error-prone responses. This led to the widely accepted view that SAT affects only decision processes, and spares more perceptual and motor stages (Ratcliff \& Rouder, 1998; Reddi \& Carpenter, 2000; Bogacz et al., 2009). This point of view was further reinforced by several fMRI studies (Ivanoff et al., 2008; Forstmann et al., 2008; van Veen et al., 2008) reporting that instructions to emphasize response speed led to an increased BOLD activity of cortical areas associated with decision processes (such as the pre-Supplementary Motor Area), but not of sensory or primary motor areas.

Recently, however, single cell recordings in monkeys showed that the rate of accumulation, as indexed by neuronal mean firing rate, is also affected by SAT (Heitz \& Schall, 2012; Hanks et al., 2014), casting doubt on a pure decision-threshold effect. Furthermore, some recent modeling studies in humans have reported that the duration of non-decision processes (represented by Ter parameter in accumulator models) sometimes needs to vary to account for the differences observed between speed and accuracy conditions (Zhang \& Rowe, 2014; Mulder et al., 2013; Dambacher \& Hübner, 2015; White et al., 2011, see also Voss et al., 2004 for a former study), suggesting a contribution of non-decision processes to SAT. However, as Ter models the compound duration of both pre- and postdecisional processes, it is impossible to determine whether sensory, motor or both processes are affected.

Few (older) studies suggested a motor locus of SAT effects. For example, it has been shown that response force is stronger when speed is emphasized (Jaskowski et al., 1994; Jaskowski et al., 2000). At the cortical level, the response-locked lateralized readiness potential $\left(\mathrm{LRP}^{1}\right)$ has been reported to be shorter under speed stress (Osman et al., 2000; Rinkenauer et al., 2004). This suggests that activity of primary motor cortices (M1s) is shorter in speed conditions, which has been interpreted as an impact of SAT on the duration of post-decisional processes. Such an interpretation, however, holds only under the assumption that M1 activation starts after decision stages have ended. This view has recently been questioned by several authors arguing that part of the decision to act is performed within the structures involved in the action execution, namely the motor structures (M1s: Donner et al., 2009; O'Connell et al., 2012;

\footnotetext{
${ }^{1}$ LRP is obtained by subtracting the mean EEG signal obtained on electrodes ipsilateral to a unimanual response from the mean signal obtained on electrodes contralateral to the response. Representing the difference of activity between the two motor cortices, it is classically considered as an index of motor preparation.
}

Kelly \& O’Connell, 2013; Frontal eye fields FEFs: Gold \& Shadlen, 2000; Purcell et al., 2010, 2012, for examples).

If at least part of the decision to act is performed within M1, the onset of response-locked LRP does not correspond to the post-decisional stage, but actually to the beginning of the action decision-making process in M1. In such a case, a SAT effect on response-locked LRP is not an argument for a motoric effect.

To clarify this point, we investigated the impact of time pressure on neurophysiological measures that unambiguously index the motor component of the reaction process. To do so, we fractioned reaction time of each trial into two sub-intervals (Burle et al., 2002) based on the responding muscles' electromyographic (EMG) activity: premotor time (PMT), from stimulus presentation until response-related EMG onset, and motor time (MT), from EMG onset to mechanical response (Fig. 1).

The MT provides a direct measure of the duration of execution processes, and has the advantage to be measurable on a trial-by-trial basis. As such, it does not suffer the distortions induced by averaging and has previously proved to be efficient to reveal modulations of late motor processes (see e.g., Possamaï et al., 2002; Burle et al., 2002; Hasbroucq et al., 1995; Tandonnet et al., 2003). To further characterize potential SAT effects on motor processes, we also examined the shape of the response EMG burst. We analyzed both the surface under the EMG burst and the slope of mean EMG profiles, respectively linked to the strength and the efficiency of the motor command.

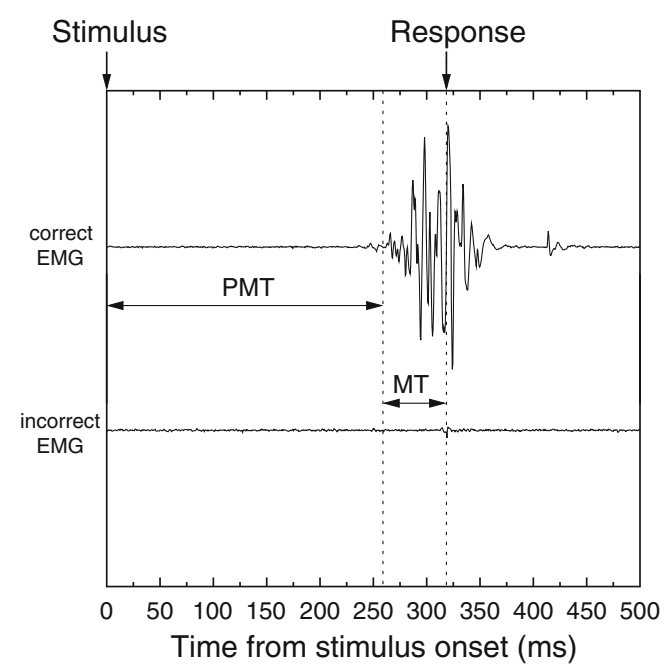

Fig. 1 EMG signal recorded during an individual trial as a function of time (stimulus occurs at time 0 ), for both the correct response hand (top), and the incorrect response hand (bottom). In each trial, premotor time is defined as the time interval between stimulus occurrence and EMG burst onset, and motor time is defined as the interval between EMG onset and response occurrence 


\section{Methods}

\section{Participants}

Sixteen participants (five women, 11 men, ages 18-50) participated in this experiment. The necessary number of participants had to be a multiple of 8 (see below for counterbalancing), and was set to 16 in the current study. All participants had normal (or corrected to normal) vision, and gave their informed consent.

\section{Apparatus}

The participants were seated in a dark room, facing a panel made of 5-digit presentation devices (model LTS-3401LP LITE ON, rise onset time $<1 \mathrm{~ms}$ ) composed of sevensegment light-emitting diodes on which the response signals (the letters "H" or "S") were presented. The whole display was contained in a $1.4^{\circ}$ visual angle. The responses were given by thumb key-presses of the right or the left hand on response micro-switch located under each response button. The electromyographic activity of the flexor pollicis brevis of both hands was recorded with two electrodes placed $2 \mathrm{~cm}$ apart on the thenar eminences. This activity was amplified, filtered (low/high-frequency cut-off at $10 \mathrm{~Hz} / 1 \mathrm{kHz}$ ), and digitized on-line (A/D rate $2 \mathrm{kHz}$ ). The EMG signal was continuously monitored by the experimenter in order to avoid any background activity that could prevent reliable detection of EMG onset. If the signal became noisy, the experimenter immediately asked the participant to relax his/her muscles. In the end, less than $2 \%$ of trials were too noisy for proper EMG detection and were excluded from analyses.

\section{Procedure}

The central digit presentation device (the target) displayed the response signal ("H" or "S"). The four other devices, flanking the target, were distractors (Eriksen \& Eriksen, 1974). Distractors were either a replication of the target ("HHHHH" or "SSSSS", compatible trials) or a replication of the alternative response signal ("HHSHH" or "SSHSS", incompatible trials). The four types of stimuli were equiprobable, and the first-order sequential effects for the trial-to-trial transition were balanced. In tasks like this, faster and more accurate responses are commonly observed in compatible compared to incompatible trials (see e.g., Kornblum et al., 1990, for a review).

Each participant took part in two experimental sessions that comprised ten blocks of 64 trials. In each session, the participants were asked to either respond very accurately, (with the cost of reaction time lengthening, "ACC" instruction) or to respond very fast (with the cost of more errors,
"SPD" instruction). SAT was thus manipulated solely by verbal instruction.

Half of the participants received the ACC instruction during the first session, whereas the other half received the SPD instruction during the first session. In each subgroup, the mapping between the target letters and the buttons was counterbalanced across participants.

\section{Evaluation of motor processes}

\section{Reaction time fractionating}

EMG traces were inspected visually and the EMG onsets were hand-scored (human pattern recognition, although more time-consuming, is superior to automated algorithms, Staude, 2001). It should be emphasized that the experimenter was unaware of the type of trial he was looking at. $^{2}$ In some trials, several EMG bursts are visible (see Burle et al., 2002, 2014 for a more detailed description of EMG trial types). In the present study, we focused on correct trials showing only the EMG burst related to the correct response (i.e., "pure-correct" trials). Based on EMG onsets, the reaction time (RT) of each pure-correct trial was fractioned into premotor time (PMT), corresponding to the interval between stimulus occurrence and EMG onset and motor time (MT), the time between the EMG onset and the response (see Fig. 1). We then analyzed the effect of speed instructions and trial compatibility, both known to impact global RT, on each of the motor and premotor time intervals.

\section{EMG signal analysis}

The impact of speed instruction on the shape of the EMG burst was investigated through the analysis of both the surface under the EMG burst and the rising slope. For each participant and each experimental condition, individual EMG activities were rectified and averaged, time locked to the EMG onset. Based on mean profiles, burst surface was determined as the surface under EMG burst in a window from EMG onset to $100 \mathrm{~ms}$ after (covering a large part of the EMG burst for all participants). The slope of the rising flank of the burst was estimated through a linear regression computed on a window from 0 to $30 \mathrm{~ms}$ after EMG onset.

\footnotetext{
${ }^{2}$ The experimenter saw the two EMG traces plotted on a computer screen, along with two vertical lines indicating the moment of the stimulus and of the response. However, no indication of the nature of the trial-i.e., no stimulus code-was provided. Besides, we also ran a fully automatic EMG onset detection, using the "EMGOnsetDetection" solution of BrainVisionAnalyser 2 (default values in the software). Although the algorithm largely over-estimated MT and inflated the within participant MT variance, because of mis-detection of the true onset, the main results were replicated, that is an effect of SAT, but not of compatibility, on MT.
} 


\section{Results}

All statistical analyses were performed by means of repeated-measures canonical analyses of variance (ANOVA), with partial eta-squared statistics $\left(\eta_{p}^{2}\right)$ reported as a measure of effect size. Percentages of errors were arcsine transformed (Winer, 1971) before being submitted to ANOVAs. For the critical results (related to potential motoric effects of SAT), these frequentist analyses were complemented by Bayesian ANOVAs using Jasp (Love et al., 2015). Such Bayesian ANOVAs provide the socalled "Bayes factor" $(B F)$, which describes the relative probability of data under competing statistical models (for example the presence or absence of an effect, see Rouder et al., $2012^{3}$ ). For all analyses, the participant was treated as random effect.

\section{Overall performance and premotor processes}

We first report overall performance, without reference to EMG data, for sake of comparison with what is usually reported. Overall mean RT and percentages of errors in each condition are shown in Table 1 . As expected, SPD instruction resulted in shorter RTs $(F(1,15)=33.35, p<$ $\left.0.001, \eta_{p}^{2}=.69\right)$ and higher error rates $(F(1,15)=$ 27.03, $\left.p<0.001, \eta_{p}^{2}=.64\right)$ compared to ACC instruction. A compatibility effect was also observed, with faster RTs $\left(F(1,15)=71.42, p<0.001, \eta_{p}^{2}=.83\right)$ and fewer errors $\left(F(1,15)=178.5, p<0.001, \eta_{p}^{2}=.92\right)$ in compatible than in incompatible trials. The interaction between the factors instruction and compatibility was not statistically significant for both RTs $\left(F(1,15)=2.47, p=0.14, \eta_{p}^{2}=\right.$ $.14)$ and error rates $\left(F(1,15)<1, p=0.79, \eta_{p}^{2}=.005\right)$.

Restricting the analysis to pure-correct trials reveals essentially the same pattern, with SAT and compatibility effects still significant on RT (respectively $F(1,15)=$ $38.23, p<0.001, \eta_{p}^{2}=.72$ and $F(1,15)=25.11, p<$ $\left.0.001, \eta_{p}^{2}=.63\right)$. The interaction proved significant in this case $\left(F(1,15)=13.84, p<0.05, \eta_{p}^{2}=.48\right)$, with the compatibility effect being larger under ACC instruction than under SPD instruction, although still significant in each condition (ACC: $21 \mathrm{~ms} ; F(1,15)=30.80, p<0.001, \eta_{p}^{2}=$ .67 ; SPD: $\left.9 \mathrm{~ms} ; F(1,15)=9.67, p<0.05, \eta_{p}^{2}=.39\right)$. Pure-correct premotor times (Table 1) showed the same pattern, being shorter under SPD than ACC instruction $\left(F(1,15)=39.38, p<0.001, \eta_{p}^{2}=.72\right)$, and shorter in compatible compared to incompatible trials $(F(1,15)=$

\footnotetext{
${ }^{3}$ The $B F$ can be reported as evidence for the $H 1$ hypothesis, and will hence be noted $B F_{10}$, or as evidence in favor of the null hypothesis $H 0$. In this case, it will be noted as $B F_{01}$. Importantly, the two are related, since $B F_{10}=\frac{1}{B F_{01}}$. Using this notation allows to always provide $B F$ superior to 1 .
}

Table 1 Top: Mean reaction times (RT) and percentages of errors for both ACC and SPD instructions, in compatible and incompatible trials

\begin{tabular}{|c|c|c|c|c|}
\hline \multicolumn{5}{|c|}{ Overall performance } \\
\hline & \multicolumn{2}{|c|}{ RT (ms) } & \multicolumn{2}{|c|}{ Errors $(\%)$} \\
\hline & $\mathrm{ACC}$ & SPD & ACC & SPD \\
\hline compatible & 547 & 440 & 5.0 & 22.7 \\
\hline incompatible & 579 & 466 & 11.6 & 36.5 \\
\hline \multicolumn{5}{|c|}{ RT fractionating (pure-correct trials) } \\
\hline & \multicolumn{2}{|c|}{ PMT (ms) } & \multicolumn{2}{|c|}{ MT (ms) } \\
\hline & $\mathrm{ACC}$ & SPD & $\mathrm{ACC}$ & SPD \\
\hline compatible & 325 & 261 & 106 & 82 \\
\hline incompatible & 346 & 269 & 106 & 84 \\
\hline
\end{tabular}

Bottom: Mean premotor times (PMT) and motor times (MT) for purecorrect trials under ACC and SPD instructions, in compatible and incompatible trials

22.11, $p<0.001, \eta_{p}^{2}=.60$ ). Again, a compatibility effect was present for the two instructions (ACC: $21 \mathrm{~ms}$; $\left.F(1,15)=31.82, p<0.001, \eta_{p}^{2}=.68\right)$; SPD: $8 \mathrm{~ms}$; $\left.F(1,15)=6.23, p<0.05, \eta_{p}^{2}=.29\right)$, but was stronger in ACC condition $\left(F(1,15)=17.11, p<0.001, \eta_{p}^{2}=.53\right)$.

\section{Evaluation of motor processes}

To investigate the impact of SAT modulations on motor processes, we first compared MT duration between the two speed instructions, and then extracted and compared the surface, as well as the rising slope of the EMG bursts.

\section{Motor time}

MTs for the different conditions are presented in Table 1. MTs were affected by speed instruction: they were $23 \mathrm{~ms}$ shorter under SPD than under ACC instruction $(F(1,15)=$ 14.13, $p<0.01, \eta_{p}^{2}=.49$ ). Compared to an effect of about $110 \mathrm{~ms}$ on the whole RT, the effect on MT hence accounts for more than $20 \%$ of the overall SAT effect.

Trial compatibility, on the other hand, had a very small numerical effect on MT ( $<1 \mathrm{~ms})$, yet marginally significant $\left(F(1,15)=3.32, p=0.09, \eta_{p}^{2}=.18\right)$. No interaction between speed instruction and compatibility was found $\left(F(1,15)=1.43, p=0.25, \eta_{p}^{2}=.09\right)$. Bayesian analyses confirmed the SAT effect on MT: the Bayes Factor $(B F)$ in favor of an effect of SAT was very high $\left(B F_{10}=247438\right)$, while it tends to favor the absence of compatibility effect on MT, although the evidence is not very strong $\left(B F_{01}=3.7\right)$. Hence, speed instruction had a clear impact on the duration of motor processes, while compatibility only had a small numerical effect, the $B F$ suggesting a slight evidence against an effect (according to current scales for interpreting $B F$, see e.g., Jeffreys, 1961). This contrasts with 
modulations of the duration of premotor processes, which were impacted by both speed instruction and compatibility.

As a SAT effect is present on both PMT and MT, we also evaluated the inter-participant correlation of the effects. This correlation was significant for both compatibility conditions $\left(r_{14}=.78, p<0.001\right.$ and $r_{14}=.66, p<0.01$, for compatible and incompatible, respectively, see left panel of Fig. 2). However, as indicated by the color code, this correlation between effects is largely driven by the overall RT of the participants.

\section{EMG burst analysis}

Mean EMG bursts obtained under each speed instruction are presented in Fig. 2 (right panel), for both compatible and incompatible trials. EMG traces clearly show that muscular activity is mainly affected by speed instruction, and not by compatibility. This was confirmed by statistical analyses showing that the EMG surface was larger $\left(F(1,15)=9.56, p<0.01, \eta_{p}^{2}=.39, B F_{10}=5840\right)$, and the burst rising slope was steeper $(F(1,15)=15.60, p<$ $\left.0.01, \eta_{p}^{2}=.51, B F_{10}=180018\right)$ under SPD compared to ACC instruction.

While compatibility showed a trend for slightly larger bursts in incompatible trials $(F(1,15)=4.22, p=$ $\left.0.06, \eta_{p}^{2}=.22\right)$, this was not supported by Bayesian analysis $\left(B F_{01}=3.897\right.$ slightly in favor of no effect), and slope analysis also supports the absence of a compatibility effect $\left(F(1,15)<1, \eta_{p}^{2}=.001, B F_{01}=3.973\right)$. No interaction between the speed instruction and the compatibility was observed (although this absence was not strongly supported by Bayesian analysis, surface: $F(1,15)<1, \eta_{p}^{2}=.03$, $B F_{01}=1.36$; slope: $F(1,15)=2.27, p=0.15$, $\left.\eta_{p}^{2}=.13, B F_{01}=1.19\right)$.

\section{Discussion}

Trading speed for accuracy is ubiquitous, present in virtually all cognitive processing and can also be evidenced in a large range of animal species, from insects to humans (see Heitz, 2014 for a recent overview). As such, SAT appears to be a core property of information processing and has recently became a de factoé critical benchmark for decision-making models. Providing an adequate description of the mechanisms underlying SAT hence seems essential for our understanding of cognition. Based on the modeling literature, displacements along the speed-accuracy tradeoff are generally assumed to be driven only by variations in the response threshold level (i.e., the amount of evidence required to trigger the response, Bogacz et al., 2009). However, recent data, both in monkeys and humans, have challenged this pure threshold-related explanation, showing that the rate of information accumulation is also affected by SAT (see Heitz, 2014 for a recent overview). In the present study, we show that non-decisional, motor-execution-related components of the reaction are also affected by SAT, contributing to more than $20 \%$ of the total effect. Importantly, although a SAT effect on motor processes duration has previously been suggested by LRP studies, these results might not be conclusive as recent data suggest that the decision to act is performed, at least in part, within the motor structures (see e.g., Donner et al., 2009; Purcell et al., 2012).

The present results, on the other hand, unambiguously establish that late motor processes also take part to RT shortening under speed stress. Such a motoric effect shows that even response execution stages can be affected by cognitive and strategic factors, indicating the necessity to consider motor components, which are often neglected, in all experimental tasks. Although not entirely incompatible, the
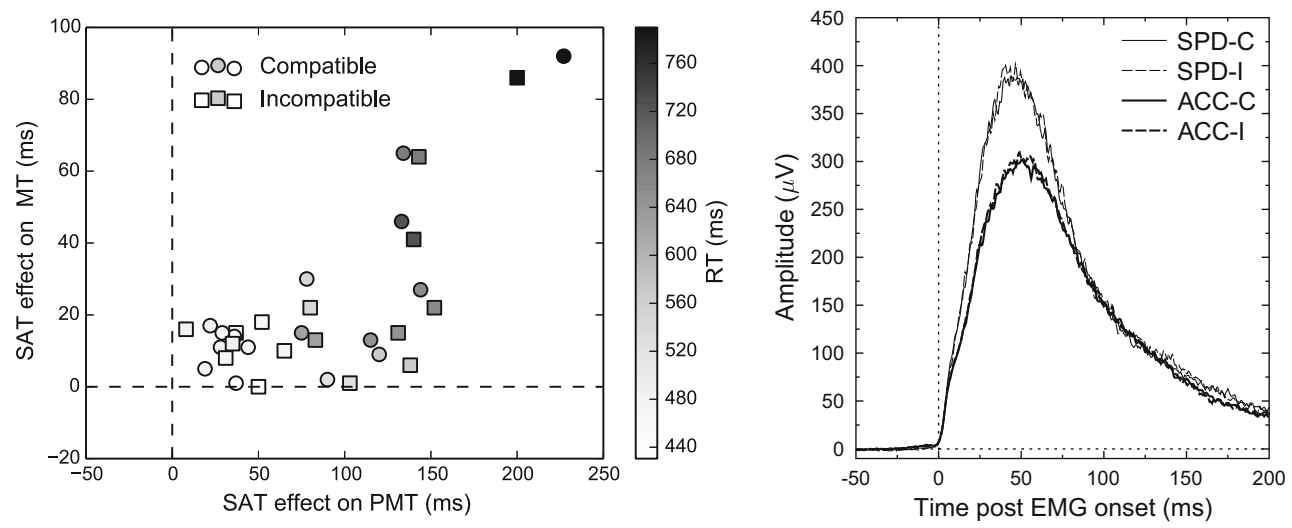

Fig. 2 Left: SAT effect on MT as a function of the SAT effect on PMT for all participants, for both compatible (circles) and incompatible (squares) conditions. The color code of the symbols corresponds to the mean RT of the participant in each compatibility condition. It appears

that the correlation is largely driven by the mean RT. Right: Mean EMG burst for both ACC and SPD instructions, in compatible and incompatible trials (black: ACC, grey: SPD ; solid line: compatible, dotted line: incompatible) 
current findings also question the assumption that responserelated and decision processes are independent, and purely serial (Calderon et al., 2015; Servant et al., 2015). ${ }^{4}$

Besides chronometry, analysis of EMG bursts further allowed for a clarification of the underlying physiological processes. In the present data, EMG bursts were shown to have larger surfaces and steeper slopes when response speed was emphasized. Steeper EMG signals index a better synchronization of the motor units discharges (Meijers et al., 1976; Ulrich \& Wing, 1991), reflecting a more efficient motor command. Hence, the faster response execution observed under SPD instruction is due to a stronger and more efficient cortical motor command.

Improving execution processes comes at a cost, however: it impairs on-line correction processes, as suggested by recent results on "partial error" trials (sub-threshold incorrect muscular activity involving within-trial correction processes-Burle et al., 2002; Spieser et al., 2015). Indeed, the efficiency to prevent "partial errors" to turn into overt errors has been shown to be reduced under time pressure (Burle et al., 2014). This might be linked to recent studies showing that the decision process continues even after a motor command has been sent (Resulaj et al., 2009; Freeman et al., 2011; Calderon et al., 2015; Servant et al., 2015; van den Berg et al., 2016), which allows to correct initial incorrect actions. However, speeding up response execution reduces the time available for a correction, thereby directly impacting correction efficiency. This hypothesis is further supported by the recent observation that participants with longer motor times present more corrected incorrect response activations (Servant et al., 2015). As a consequence, SAT also occurs within the motor execution level, contributing to the global trade-off both in terms of chronometry and of error rate, as speeding up motor execution increases the number of overt (uncorrected) errors. SAT effects on premotor and motor components appear correlated, and covary with reaction time, raising the possibility of a common origin. The common denominator could be our observed faster buildup rate of EMG activity and the recent observation that the rate of information accumulation in the decision stage is faster under speed than under accurate instruction (Heitz \& Schall, 2012; Hanks et al., 2014), paralleling the steeper EMG burst observed in the present data. Whether the rate of EMG recruitment directly depends on the information accumulation rate remains an open question.

To conclude, the present results unambiguously demonstrate that motor components of the reaction processes are not spared by SAT. Not only are they affected by time pressure but they also largely contribute to this trade-off, since

\footnotetext{
${ }^{4} \mathrm{~A}$ single factor can affect two serial stages independently
}

shortening motor execution stages also increases the number of overt errors by reducing the likelihood of interrupting and correcting incorrect response activation (Burle et al., 2014; Servant et al., 2015). This confirms, in humans, that SAT is a more diverse effect than a simple modulation of threshold level, as also recently argued (Heitz \& Schall, 2012).

Acknowledgments This research was supported by the European Research Council under the European Community's Seventh Framework Program (FP7/2007-2013 Grant Agreement no. 241077). The authors wish to thank Hidekazu Kaneko for his help during the early stages of this project.

\section{References}

Bogacz, R., Wagenmakers, E. J., Forstmann, B. U., \& Nieuwenhuis, S. (2009). The neural basis of the speed-accuracy tradeoff. Trends in Neurosciences, 33, 10-16.

Brunton, B. W., Botvinick, M. M., \& Brody, C. D. (2013). Rats and humans can optimally accumulate evidence for decision-making. Science, 340(6128), 95-98. doi:10.1126/science. 1233912

Burle, B., Possamaï, C.A., Vidal, F., Bonnet, M., \& Hasbroucq, T. (2002). Executive control in the Simon effect: an electromyographic and distributional analysis. Psychological Research Psychologische Forschung, 66, 324-336.

Burle, B., Spieser, L., Servant, M., \& Hasbroucq, T. (2014). Distributional reaction time properties in the Eriksen task: marked differences or hidden similarities with the Simon task? Psychonomic Bulletin \& Review, 21(4), 1003-1010. doi:10.3758/s13423-013-0561-6

Calderon, C. B., Verguts, T., \& Gevers, W. (2015). Losing the boundary: cognition biases action well after action selection. Journal of Experimental Psychology: General, 144(4), 737-743. doi:10.1037/xge0000087

Dambacher, M., \& Hübner, R. (2015). Time pressure affects the efficiency of perceptual processing in decisions under conflict. Psychological Research Psychologische Forschung, 79(1), 83-94. doi:10.1007/s00426-014-0542-z

DasGupta, S., Ferreira, C. H., \& Miesenböck, G. (2014). Foxp influences the speed and accuracy of a perceptual decision in Drosophila. Science, 344(6186), 901-904. doi:10.1126/science.1252114.

Donner, T. H., Siegel, M., Fries, P., \& Engel, A. K. (2009). Buildup of choice-predictive activity in human motor cortex during perceptual decision making. Current Biology, 19, 1581-1585.

Eriksen, B. A., \& Eriksen, C. W. (1974). Effects of noise letters upon the identification of target letter in a non-search task. Perception \& Psychophysics, 16, 143-149.

Forstmann, B. U., Dutilh, G., Brown, S., Neumann, J., von Cramon, D. Y., Ridderinkhof, K. R., \& Wagenmakers, E. J. (2008). Striatum and pre-SMA facilitate decision-making under time pressure. Proceedings of the National Academy of Sciences, 105(45), 17538-17542. doi:10.1073/pnas.0805903105

Freeman, J. B., Dale, R., \& Farmer, T. A. (2011). Hand in motion reveals mind in motion. Frontiers in Psychology, 2, 59. doi:10.3389/fpsyg.2011.00059

Gold, J. I., \& Shadlen, M. N. (2000). Representation of a perceptual decision in developing oculomotor commands. Nature, 404, 390394.

Hanks, T., Kiani, R., \& Shadlen, M. N. (2014). A neural mechanism of speed-accuracy tradeoff in macaque area lip. eLife, 3, e02260. 
Hasbroucq, T., Akamatsu, M., Mouret, I., \& Seal, J. (1995). Fingers pairings in two-choice reaction time tasks: does the between hands advantage reflect response preparation. Journal of Motor Behavior, 27, 251-262.

Heitz, R. P. (2014). The speed-accuracy tradeoff: history, physiology, methodology, and behavior. Frontiers in Neuroscience, 8, 150. doi:10.3389/fnins.2014.00150

Heitz, R. P., \& Schall, J. D. (2012). Neural mechanisms of speedaccuracy tradeoff. Neuron, 76, 616-628.

Ivanoff, J., Branning, P., \& Marois, R. (2008). FMRI evidence for a dual process account of the speed-accuracy tradeoff in decisionmaking. PLOS ONE, 3, e2635.

Jaskowski, P., van der Lubbe, R. H., Wauschkuhn, B., Wascher, E., \& Verleger, R. (2000). The influence of time pressure and cue validity on response force in an s1-s2 paradigm. Acta Psychologica, 105(1), 89-105.

Jaskowski, P., Verleger, R., \& Wascher, E. (1994). Response force and reaction time in a simple reaction task under time pressure. Zeitschrift für Psychologie mit Zeitschrift fü,r agewandte Psychologie, 202(4), 405-413.

Jeffreys, H. (1961). Theory of probability. Oxford: Oxford University Press.

Kelly, S. P., \& O'Connell, R. G. (2013). Internal and external influences on the rate of sensory evidence accumulation in the human brain. Journal of Neuroscience, 33, 19434-19441.

Kornblum, S., Hasbroucq, T., \& Osman, A. (1990). Dimensional overlap: cognitive basis for stimulus-response compatibilitya model and taxonomy. Psychological Review, 97(2), 253270.

Love, J., Selker, R., Marsman, M., Jamil, T., Dropmann, D., Verhagen, A. J., ..., \& Wagenmakers, E. J. (2015). Jasp (version 0.7) (computer software). https://jasp-stats.org/

Meijers, L. M., Teulings, J. L., \& Eijkman, E. G. (1976). Model of the electromyographic activity during brief isometric contractions. Biological Cybernetics, 25(1), 7-16.

Mulder, M. J., Keuken, M. C., van Maanen, L., Boekel, W., Forstmann, B. U., \& Wagenmakers, E. J. (2013). The speed and accuracy of perceptual decisions in a random-tone pitch task. Attention, Perception, and Psychophysics, 75(5), 1048-1058. doi:10.3758/s13414-013-0447-8

O'Connell, R. G., Dockree, P. M., \& Kelly, S. P. (2012). A supramodal accumulation-to-bound signal that determines perceptual decisions in humans. Nature Neuroscience, 15, 1729-1735.

Osman, A., Lianggang, L., Müller-Gethmann, H., Rinkenauer, G., Mattes, S., \& Ulrich, R. (2000). Mechanisms of speed-accuracy tradeoff: evidence from covert motor processes. Biological Psychiatry, 51, 173-199.

Possamaï, C.A., Burle, B., Osman, A., \& Hasbroucq, T. (2002). Partial advance information, number of alternatives, and motor processes: an electromyographic study. Acta Psychologica, 111, 125139.

Purcell, B. A., Heitz, R. P., Cohen, J. Y., Schall, J. D., Logan, G. D., \& Palmeri, T. J. (2010). Neurally constrained modeling of perceptual decision making. Psychological Review, 117, 1113-1143.

Purcell, B. A., Schall, J. D., Logan, G. D., \& Palmeri, T. J. (2012). From salience to saccades: multiple-alternative gated stochastic accumulator model of visual search. Journal of Neuroscience, 32 , 3433-3446.
Ratcliff, R., \& Rouder, J. N. (1998). Modeling response times for twochoice decisions. Psychological Science, 9, 347-356.

Ratcliff, R., \& Smith, P. L. (2004). A comparison of sequential sampling models for two-choice reaction time. Psychological Review, 111(2), 333-367. doi:10.1037/0033-295X.111.2.333

Reddi, B. A. J., \& Carpenter, R. H. S. (2000). The influence of urgency on decision time. Nature Neuroscience, 3, 827-830.

Resulaj, A., Kiani, R., Wolpert, D. M., \& Shadlen, M. N. (2009). Changes of mind in decision-making. Nature, 461(7261), 263266. doi:10.1038/nature 08275

Rinkenauer, G., Osman, A., Ulrich, R., Müller-Gethmann, H., \& Mattes, S. (2004). On the locus of speed-accuracy trade-off in reaction time: inferences from the lateralized readiness potential. Journal of Experimental Psychology: General, 133, 261-282.

Rouder, J., Morey, R. D., Speckman, P., \& Province, J. M. (2012). Default Bayes factors for ANOVA designs. Journal of Mathematical Psychology, 56, 356-374.

Servant, M., White, C., Montagnini, A., \& Burle, B. (2015). Using covert response activation to test latent assumptions of formal decision-making models. The Journal of Neuroscience, 35, 10371-10385.

Spieser, L., van den Wildenberg, W., Hasbroucq, T., Ridderinkhof, K. R., \& Burle, B. (2015). Controlling your impulses: electrical stimulation of the human supplementary motor complex prevents impulsive errors. The Journal of Neuroscience, 35(7), 3010-3015. doi:10.1523/JNEUROSCI.1642-14.2015

Staude, G. H. (2001). Precise onset detection of human motor responses using a whitening filter and the log-likelihood-ratio test. IEEE Transactions on Biomedical Engineering, 48(11), 12921305. doi: $10.1109 / 10.959325$

Tandonnet, C., Burle, B., Vidal, F., \& Hasbroucq, T. (2003). The influence of time preparation on motor processes assessed by surface Laplacian estimation. Clinical Neurophysiology, 114, 2376-2384.

Ulrich, R., \& Wing, A. M. (1991). A recruitment theory of force-time relations in the production of brief force pulses: the parallel force unit model. Psychological Review, 98(2), 268-294.

van den Berg, R., Anandalingam, K., Zylberberg, A., Kiani, R., Shadlen, M. N., \& Wolpert, D. M. (2016). A common mechanism underlies changes of mind about decisions and confidence. Elife, 5. doi:10.7554/eLife. 12192

van Veen, V., Krug, M. K., \& Carter, C. S. (2008). The neural and computational basis of controlled speed-accuracy tradeoff during task performance. Journal of Cognitive Neuroscience, 20(11), 1952-1965. doi:10.1162/jocn.2008.20146

Voss, A., Rothermund, K., \& Voss, J. (2004). Interpreting the parameters of the diffusion model: an empirical validation. Memory \& Cognition, 32(7), 1206-1220.

White, C. N., Ratcliff, R., \& Starns, J. J. (2011). Diffusion models of the flanker task: discrete versus gradual attentional selection. Cognitive Psychology, 63(4), 210-238. doi:10.1016/j.cogpsych.2011.08.001

Winer, B. J. (1971). Statistical principles in experimental design: design and analysis of factorial experiments. New York: McGrawHill.

Zhang, J., \& Rowe, J. B. (2014). Dissociable mechanisms of speedaccuracy tradeoff during visual perceptual learning are revealed by a hierarchical drift-diffusion model. Frontiers in Neuroscience, 8 , 69. doi:10.3389/fnins.2014.00069 